The Search for Reconnection and Helicity During Formation of a Bounded Spheromak

C. A. Romero-Talamas, H. S. McLean, E. B. Hooper, R. D. Wood, L. L. LoDestro, J. M. Moller

April 30, 2007

Journal of Fusion Energy 
This document was prepared as an account of work sponsored by an agency of the United States Government. Neither the United States Government nor the University of California nor any of their employees, makes any warranty, express or implied, or assumes any legal liability or responsibility for the accuracy, completeness, or usefulness of any information, apparatus, product, or process disclosed, or represents that its use would not infringe privately owned rights. Reference herein to any specific commercial product, process, or service by trade name, trademark, manufacturer, or otherwise, does not necessarily constitute or imply its endorsement, recommendation, or favoring by the United States Government or the University of California. The views and opinions of authors expressed herein do not necessarily state or reflect those of the United States Government or the University of California, and shall not be used for advertising or product endorsement purposes. 


\title{
The search for reconnection and helicity during formation of a bounded spheromak
}

\author{
C. A. Romero-Talamás, ${ }^{*}$ H. S. McLean, E. B. Hooper, \\ R. D. Wood, L. L. LoDestro, and J. M. Moller \\ Lawrence Livermore National Laboratory, \\ 7000 East Avenue, Livermore, California, 94550.
}

(Dated: April 27, 2007)

\begin{abstract}
Recent results from investigations using insertable magnetic probes at the Sustained Spheromak Physics Experiment (SSPX) [E. B. Hooper et al., Nucl. Fusion 39, 863 (1999)] are presented. Experiments were carried out during pre-programmed, constant amplitude coaxial gun current pulses, where magnetic field increases stepwise with every pulse, but eventually saturates. Magnetic traces from the probe, which is electrically isolated from the plasma and spans the flux conserver radius, indicate there is a time lag at every pulse between the response to the current rise in the open flux surfaces (intercepting the electrodes) and the closed flux surfaces (linked around the open ones). This is interpreted as the time to buildup enough helicity in the open flux surfaces before reconnecting and merging with the closed ones. Future experimental and diagnostic plans to directly estimate the helicity in the open flux surfaces and measure reconnection are briefly discussed.
\end{abstract}

\footnotetext{
*Electronic address: romerotalamas1@ilnl.gov

$\dagger$ This work performed under the auspices of the USDOE by LLNL under contract 7405-Eng-48. The authors are grateful to Paul M. Bellan and Caltech for their continued support to SSPX with the high-speed imaging hardware, and to the Center for Magnetic Self-Organization in Laboratory and Astrophysical Plasmas (CMSO) for their continued financial support.
} 


\section{INTRODUCTION}

The spheromak is a toroidal confinement scheme with the potential for containing fusion plasmas. The volume in which spheromaks are produced is simply connected (no external coils linking the plasma), but currents in the plasma self-organize to form both open and closed magnetic surfaces that can be sustained by means of coaxial helicity injection (CHI) [1]. The CHI method uses a pre-programed magnetic field which is initially open (intercepting the chamber volume), and serves as a guiding field once current starts flowing between the CHI gun electrodes. This current adds helicity to the magnetic field, which eventually reconnects and relaxes to the so-called Taylor state [2] to form the linked field that constitute the spheromak. However, the process of going from open to closed surfaces is not well understood. In particular, it is not understood where and when reconnection occurs, and if the process can be optimized in order to maximize the strength of the closed flux surfaces in order to effectively contain high-temperature plasmas.

Investigations at the Sustained Spheromak Physics Experiment [3] are being conducted with insertable magnetic probes to help understand the spheromak formation and magnetic field buildup process. The initial breakdown and plasma ejection process has been previously presented in Refs. [4-6] and is the subject of ongoing research. Here, however, we focus on the most recent experiments of repeated gun current pulses which build flux in a stepwise fashion at every pulse. The experimental setup and recent measurements are presented in the section below, and a brief discussion and plans for follow up experiments are presented in Section III.

\section{EXPERIMENTS AND MEASUREMENTS}

The recently installed modular capacitor bank was programmed to produce a series of gun current pulses, such as the $I_{g}$ trace shown in Fig. 1. The first pulse is called the formation pulse. It is during this pulse that plasma breaks down and the first closed surfaces appear. However, strong field asymmetries lead to poor confinement, high plasma resistivity, and a rapid magnetic field decay. Asymmetries are also found before closed flux appear, which occur in the first $100 \mu s$ of the shot. Typical open flux surface asymmetries are shown in Fig. 2. The plasma structures in these images have not developed closed flux, and 
evolve from an initially vertical plasma column conducting hundreds of kilo-amperes. It is conjectured that the strong kinks seen in these images evolve to acquire the same or more helicity as a linked structure, similarly to the flux rope model depicted at the left of Fig. 2 , before reconnecting and forming the closed flux that are inferred shortly after the kink. Unfortunately, high-speed images cannot follow the entire process as only two images per plasma shot are possible and plasma emission falls outside the camera range at this time. Magnetic probes are thus used to follow this formation process.

Numerical reconstructions of magnetic surfaces using the two-dimensional magnetohydrodynamic code CORSICA [7], which uses a number of edge magnetic probes to set boundary conditions, are also difficult during the initial pulse since the aforementioned field asymmetries induce large errors in the reconstruction. (For flat current profiles and low amplitude toroidal modes, uncertainties in CORSICA reconstructions are small.)

For the subsequent current pulses the conditions are not the same as for the first pulse, both for shots with and without the insertable probe. Although plasma is very resistive compared to shots that do not have the probe inserted, subsequent pulses have a linked topology and magnetic energy does not disappear completely between pulses. In fact, magnetic field builds with every pulse along the entire flux conserver radius. For shots that do not have the probe inserted, or "clean shots", the magnetic field measured at the edge probes also increases stepwise with every pulse, but decays much slower than in shots where the probe is inserted.

Closer inspection on the probe signals during any pulse after formation reveal that the magnetic field does not respond instantly to the increase in current everywhere in the spheromak. Along the open flux surfaces, the magnetic field does respond instantly. However, where closed surfaces are expected, the field takes approximately $120 \mu s$ to respond (i.e., when $d B / d t$ changes sign), as shown in Fig. 3. Moreover, at this response time there is also a small decrease in the magnetic energy in the open flux surfaces.

\section{DISCUSSION}

The simultaneous magnetic field response to current increase in the open and closed flux surfaces, in the pulses after the formation pulse, is conjectured to be the time at which

poloidal current is transfered to toroidal current via magnetic reconnection and merging. 
This event is depicted schematically in Fig. 3(a), and is an extension of the flux rope model shown in Fig 2. In this simple flux rope model, the open flux surfaces acquire helicity as the current increases until it has enough to kink and reconnect, forming stronger closed flux surfaces at each pulse.

An indication that flux in these shots builds up through reconnection, and not simply through diffusion, is the time it takes for the magnetic field to respond at different locations along the probe. A rough estimate of the magnetic diffusion through the plasma can be done using dimensional analysis in planar geometry. Magnetic diffusion through a conducting surface is estimated from $d \mathbf{B} / d t=\alpha \nabla^{2} \mathbf{B}$, which is obtained by combining $\nabla \times \mathbf{B}=\mu_{o} \mathbf{J}$, $\nabla \times \mathbf{E}=-\partial \mathbf{B} / \partial t$, and $\mathbf{E}=\eta \mathbf{J}$, and where $\alpha=\eta / \mu_{o}$. For shots with the probe inserted, electron temperature has been measured using Thomson scattering [8] at $20 \mathrm{eV}$. Using Sptizer resistivity [9] to estimate $\eta$ (with $Z_{\text {eff }}=2$ and $\ln \Lambda \sim 10$ ), the order of magnitude diffusion time between probe $|B|_{7}$ to probe $|B|_{19}(24 \mathrm{~cm})$ in Fig. 1 is $\tau \sim 3 \mathrm{~ms}$. This time is much longer than what is observed in the magnetic traces.

The helicity input rate is modeled for coaxial guns by the expression [6] $d K / d t=2 V_{g} \Psi_{g}-$ $2 \int \eta \mathbf{J} \cdot \mathbf{B} d^{3} r$, where $\eta$ is the plasma's resistivity, and $\Psi_{g}$ is the gun flux (fixed at SSPX for the duration of a plasma shot). It is clear from the gun voltage trace in Fig. 1 that the helicity input rate increases at the beginning of every pulse, given the average increase in $V_{g}$, and then decreases as the current ramps down.

In terms of the flux rope model, it is to be expected that as the magnetic field in the closed flux surfaces becomes stronger after each reconnection event, it also becomes increasingly difficult for the open flux surfaces to kink and acquire the minimum amount of helicity in order to reconnect. For a pulsed current profile with fixed amplitude, this translates to saturation of the magnetic field. This saturation is indicated in Fig. 1 by a thick dashed line on the signal closest to the geometric axis.

Future research plans include new experiments where the $I_{g}$ amplitude increases at each pulse. The construction of a new insertable magnetic probe is also underway. This probe will have two rows of inductor clusters that will be placed inside the plasma and will be used primarily to measure the open magnetic flux evolution (i.e., kinking) during formation and the subsequent pulses. These measurements will be compared with computer models of kinked current-carrying ropes that include synthetic magnetic probes. A schematic of the probe under construction and computer model flux rope measurements are shown in Fig. 4. 
The probe will also be used to measure reconnection if it occurs in the area delimited by the two stems. Details probe construction and of the computer models used in the signal analysis will be reported elsewhere.

[1] T. R. Jarboe. Plasma Phys. Control. Fusion, 36:945, 1994.

[2] J. B. Taylor. Phys. Rev. Lett., 33:1139, 1974.

[3] E. B. Hooper, L. D. Pearlstein, and R. H. Bulmer. Nucl. Fusion, 39:863, 1999.

[4] C. A. Romero-Talamás, E. B. Hooper, D. N. Hill, B. I. Cohen, H. S. McLean, R. D. Wood, and J. M. Moller. J. Fusion Energy, 26:169, 2007.

[5] C. A. Romero-Talamás, C. Holcomb, P. M. Bellan, and D. N. Hill. Phys. Plasmas, 13(12):022502, 2006 .

[6] S. Woodruff, B. I. Cohen, E. B. Hooper, H. S. McLean, B. W. Stallard, D. N. Hill, C. T. Holcomb, C. Romero-Talamas, R. D. Wood, G. Cone, and C. R. Sovinec. Phys. Plasmas, 12:052502, 2005.

[7] J. A. Crotinger, L. LoDestro, L. D. Pearlstein, A. Tarditi, T. A. Casper, and E. B. Hooper. Corsica: A comprehensive simulation of toroidal magnetic-fusion devices. final report to the ldrd program. Technical report, Lawrence Livermore National Laboratory, 1997.

[8] H. S. McLean, A. Ahmed, D. Buchenauer, et al. Rev. Sci. Instrum., 72:556, 2001.

[9] J. D. Huba. NRL Plasma Formulary. Naval Research Laboratory, Washington, 2002. p.p. $28-29$.

[10] C. A. Romero-Talamás, P. M. Bellan, and S. C. Hsu. Rev. Sci. Instrum., 75:2664, 2004.

This work was performed under the auspices of the U.S. Department of Energy by University of California, Lawrence Livermore National Laboratory under Contract W-7405-Eng-48. 


\section{FIGURE CAPTIONS}

- Figure 1. Left: shot 16209. Traces are, from top to bottom: gun voltage $V_{g}$; gun current $I_{g}$; magnitude of the magnetic field $\left(|B|=\sqrt{B_{r}^{2}+B_{\theta}^{2}+B_{z}^{2}}\right)$ at probe clusters 1 through 19 and a poloidal wall probe, as indicated in the schematic. Right: Typical CORSICA reconstruction for SSPX magnetic flux surfaces during flat current profiles of similar magnitude as the one shown here; the position of the insertable probe and its clusters is overlaid on the contours.

- Figure 2. Left: Flux rope model in which open flux surfaces acquire enough helicity to reconnect and form a linked structure with the same helicity. Right: SSPX sample images of kinked open flux surfaces shortly after plasma breakdown and ejection from the gun. These type of images inspired the application of the flux rope model to SSPX plasmas (see Ref. [5] and references therein).

- Figure 3. (a) Flux rope model for multiple current pulses. (b) Close-up of Fig. 1. The first vertical dashed line shows when the current pulse starts. The second vertical dashed line shows when the closed flux responds to the current rise.

- Figure 4. Concept of double insertable probe and its movable mechanism. The probe is designed to be inserted through the diagnostics slot in the SSPX flux conserver, and then open as shown. The mechanism is made of a combination of metallic and ceramic parts to avoid arcing or welding during strong electromagnetic pulses. Each probe stem is similar in construction to the probe reported in Ref. [10]. 

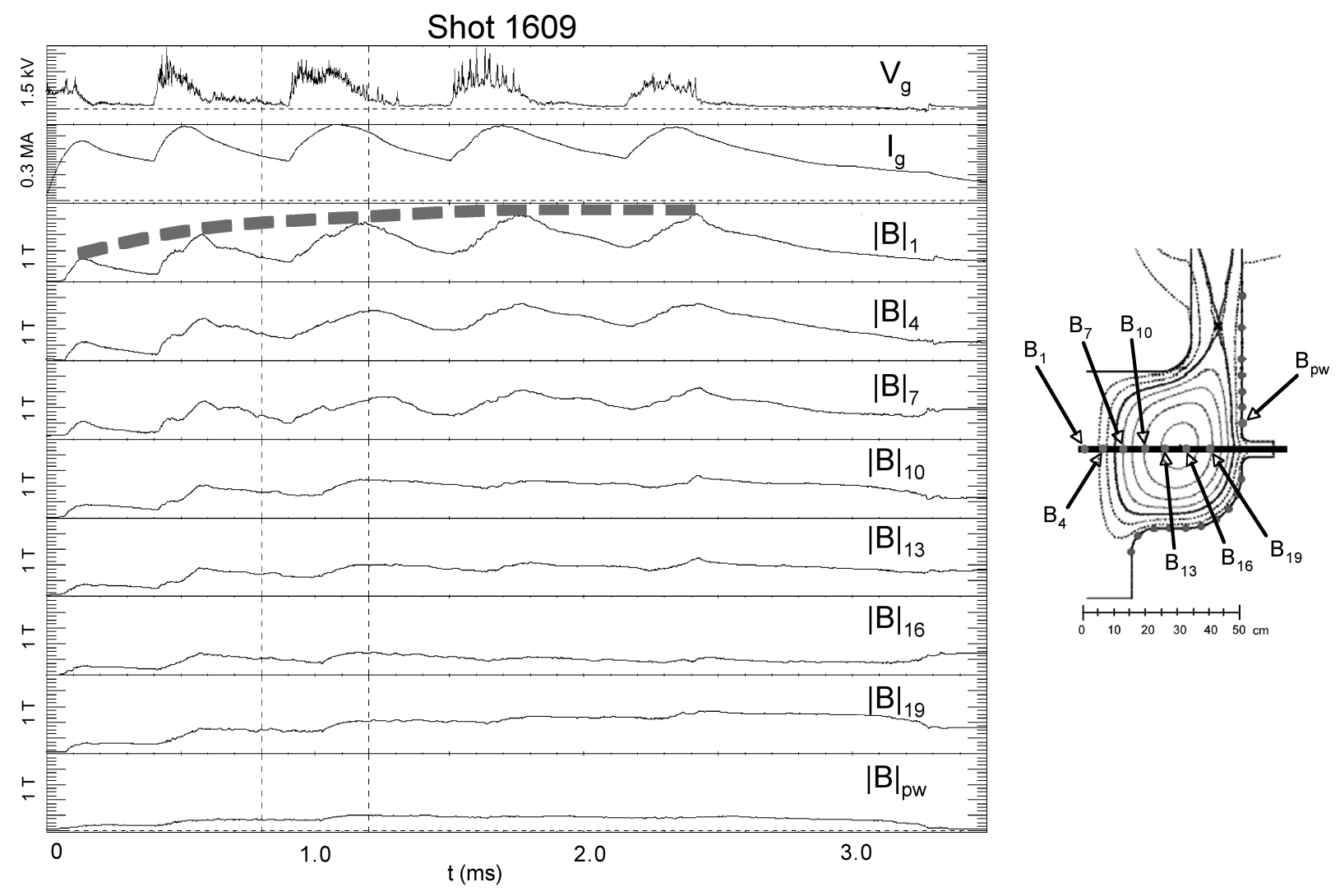

FIG. 1: 



FIG. 2: 

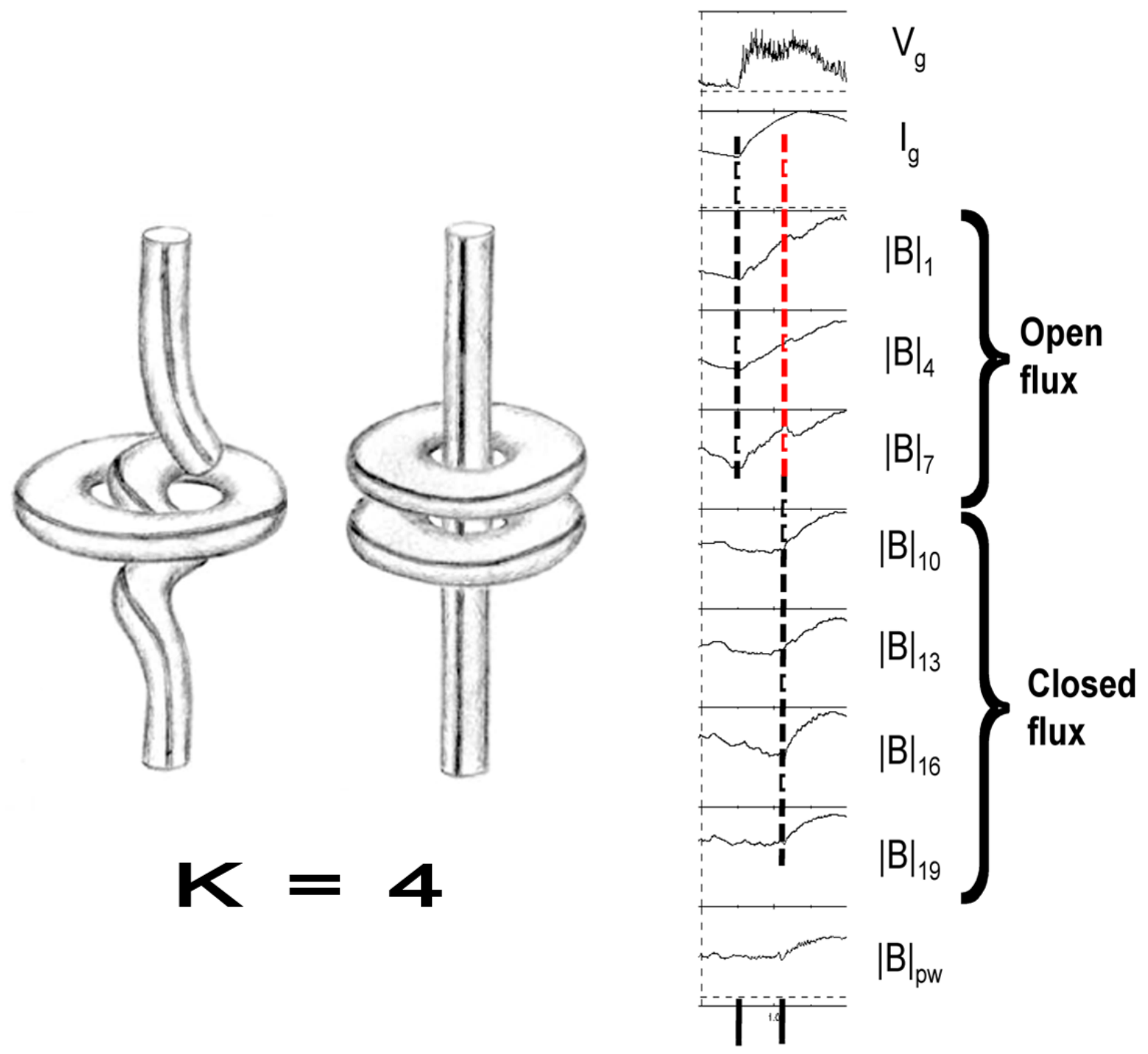

$\Delta \mathrm{t} \sim 120 \mu \mathrm{s}$

(a)

(b)

FIG. 3: 

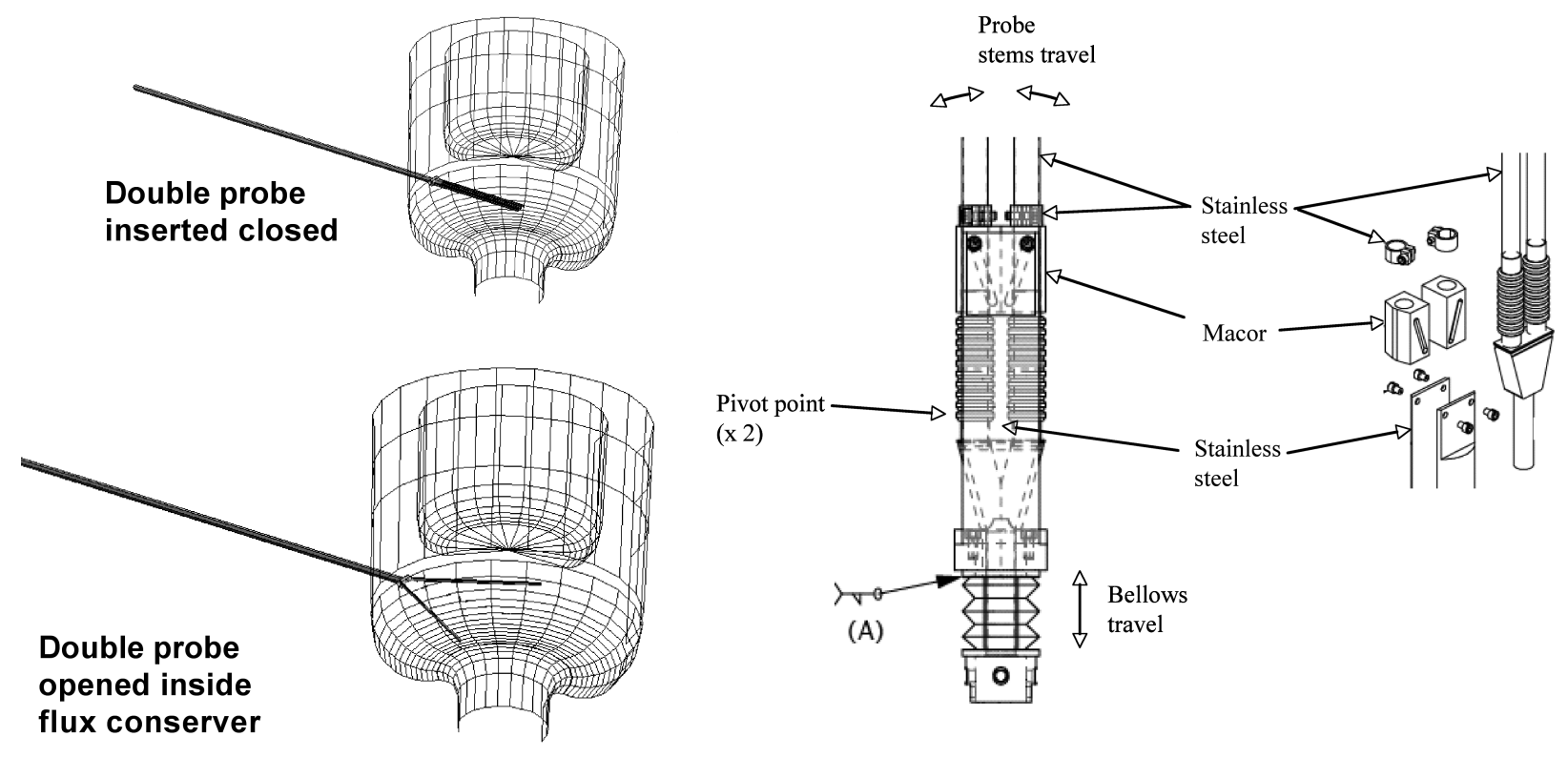

FIG. 4: 